\title{
SEISMICITY IN FINLAND AGAINST A BACKGROUND OF THE COSMIC CONDITIONS
}

\author{
G. P. TAMrazyan
}

Institute of Geology, Academy of Sciences of the Azerbaijanian SSR

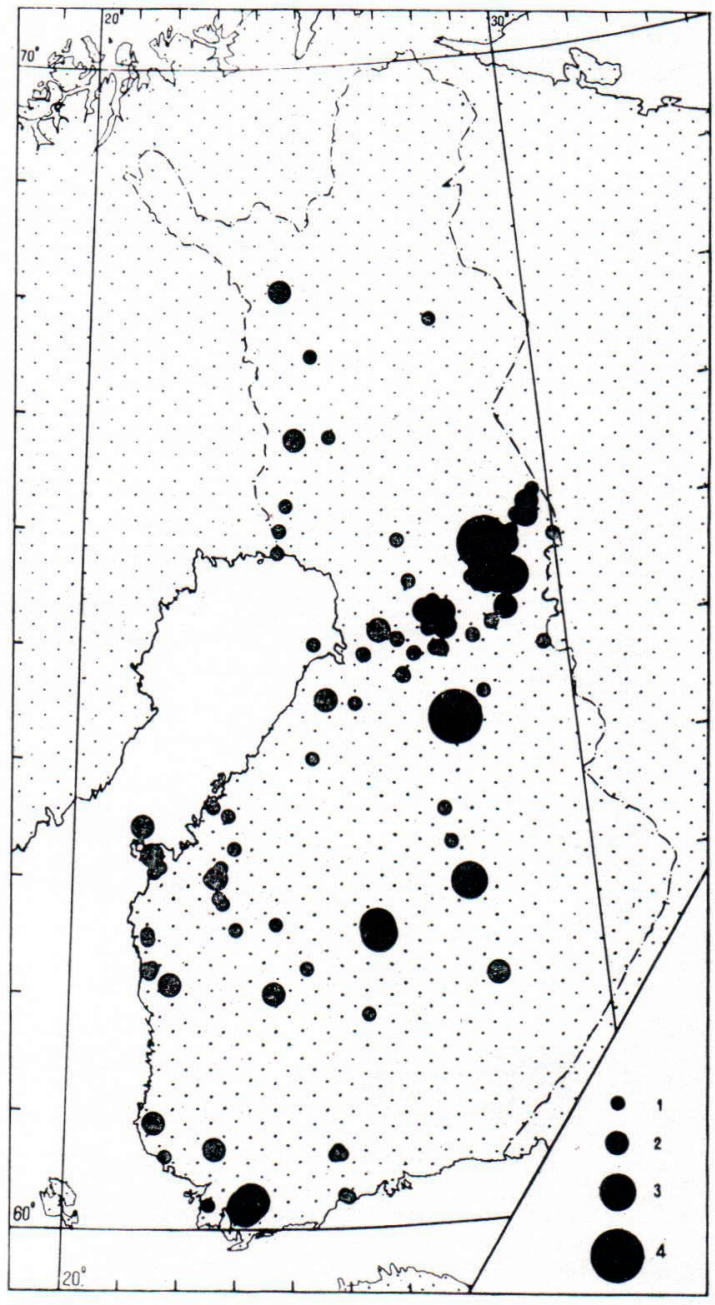

Fig. 1. Finland and earthquakes within its limits (19001950 ). The magnitudes (M) of the earthquakes are: 1 ) $2.1-3.0$; 2) $3.1-4.0$; 3) $4.1-5.0$; 4) $5.1-5.2$.
Earthquakes of various plicated regions of the world proved to be dependent on cosmic conditions in which the Earth is (Tamrazyan 1967, 1966). The platform regions and the shield area in particular have been insufficiently studied in this respect. Some of the problems concerning earthquake distribution under various cosmic conditions conform to the very old shield area of world, the Baltic shield, which comes under brief consideration in this paper. The article considers seismicity in Finland in connection with cosmic conditions. Earthquakes from 1900 to 1950 will be considered according to the published works (Båth 1956). Within these 50 years (1900_1950) about a hundred earthquakes were registered (Fig. 1.).

Below, in order to analyse the seismic material, it has been necessary to use some conventional terms, such as synodic and reduced anomalistic age of the earthquakes as well as reduced lunar days.

The average synodic age of an earthquake is the time (in days) which has elapsed after the new moon prior to the earthquake and which is calculated as relative to the average duration of a synodic month consisting of 29.6 days. In this paper the difference in the time interval between two real subsequent new moons and the duration of the average synodic month is corrected.

The reduced anomalistic age of an earthquake is the time interval (in days) which has elapsed since the last passage of the moon through the 
TABLE 1

Strong earthquakes in Finland (1900-1950)

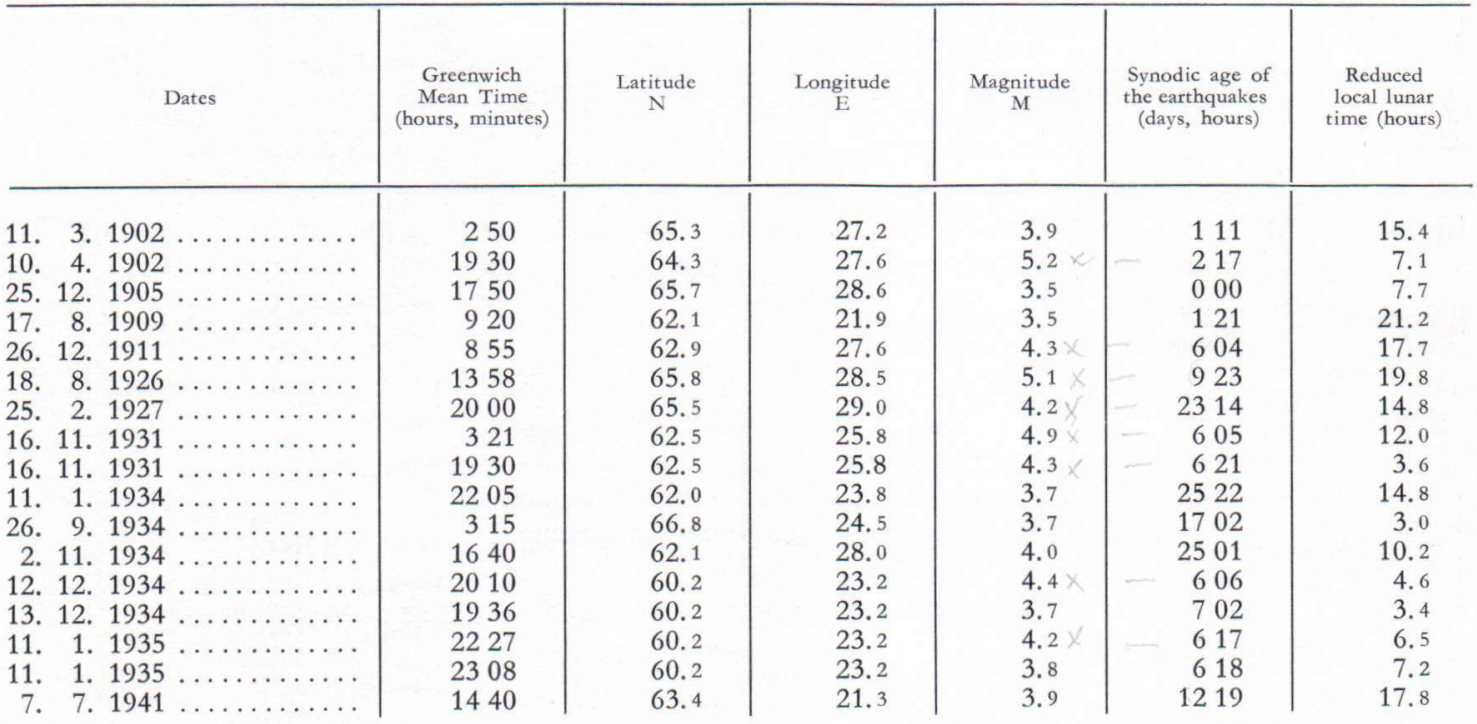

perigee, and which is calculated from the average duration of an anomalistic month of 27. 6 days. For the conversion of the current anomalistic age into the reduced anomalistic age a correction is introduced (sometimes up to some dozens of hours).

For the purpose of uniformity, it is convenient to use the reduced lunar days conjugated by duration with the solar days instead of the lunar days with a duration of 24 hours 50 minutes.

The distribution of earthquakes in Finland (with magnitude $\mathrm{M} \geqq 3$ ) according to the day of the synodic month is shown in Fig. 2. As is seen from the drawing, the strong earthquakes with a magnitude $\mathrm{M} \geqq 4$ are distributed unevenly within the synodic month. The greatest number of earthquakes is located in the interval between the 2 nd and 10 th of the synodic month (Table 1 ). Thus, e.g. out of 8 strong earthquakes less than 7 took place at the synodic age in the interval between the 2 nd and 10 th days. Thus the reciprocal location of the three interacting bodies (the Earth, the Moon and the Sun) is found to

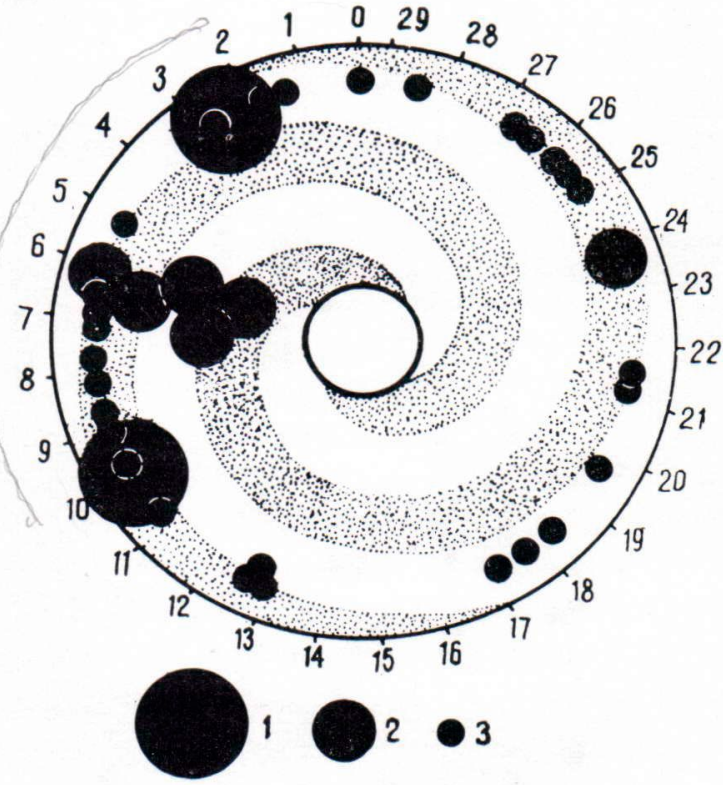

Fig. 2 Diagram of the distribution of strong earthquakes (with magnitude $M \geq 3$ ) in Finland.

Date of the average synodic month is indicated in the circumference. The magnitude $(M)$ of the earthquakes are: 1) $5.1-5.2$;2) $4.1-5.0$;3) $3.0-4.0$. 
be of some importance to the origination of earthquakes.

The distribution of seismicity according to the dates of the synodic month becomes especially selective when examining the energy released by earthquakes (Fig. 3). In Finland most seismic energy $(96 \%$ ) was released in the interval between the 2 nd and 10 th days of the synodic month. For the rest of the synodic month (1029. 6 and $0-2$ days) the daily release of energy was 56 times less than that within the forementioned days ( $2-10$ days) of the synodic month. This is a remarkable fact, indicating the extremely important role played by lunar phases in the distribution of seismic energy during the lunar month.

The distribution of seismic energy depending on the synodic and reduced anomalistic age is shown in Fig. 4. It is seen that most of the strong earthquakes (with magnitude $\mathrm{M} \geq 4.1$ ) in Finland are located in narrow bands elongated in a direction going from the left hand corner to the right upper one.

The distribution of the strong earthquakes according to local lunar time and simultaneously according to their synodic age is shown in Fig. 5 . An almost distinct maximum of the strong earthquakes in certain sections of this figure indicates the role of cosmic influences on the period of intense seismic activity in Finland.

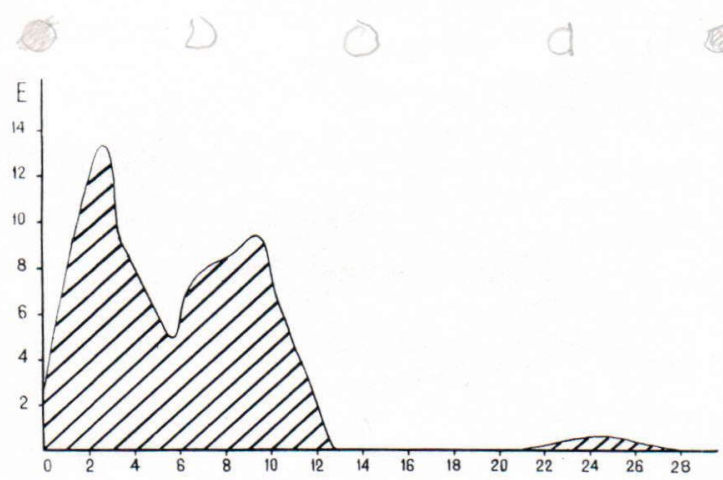

Fig. 3. Distribution of the energy of earthquakes in Finland from 1900-1950 depending on the average synodic age; the change of seismic energy $\left(\mathrm{E}, \times 10^{18} \mathrm{erg}\right)$ is shown by twice-slidding three-days period.

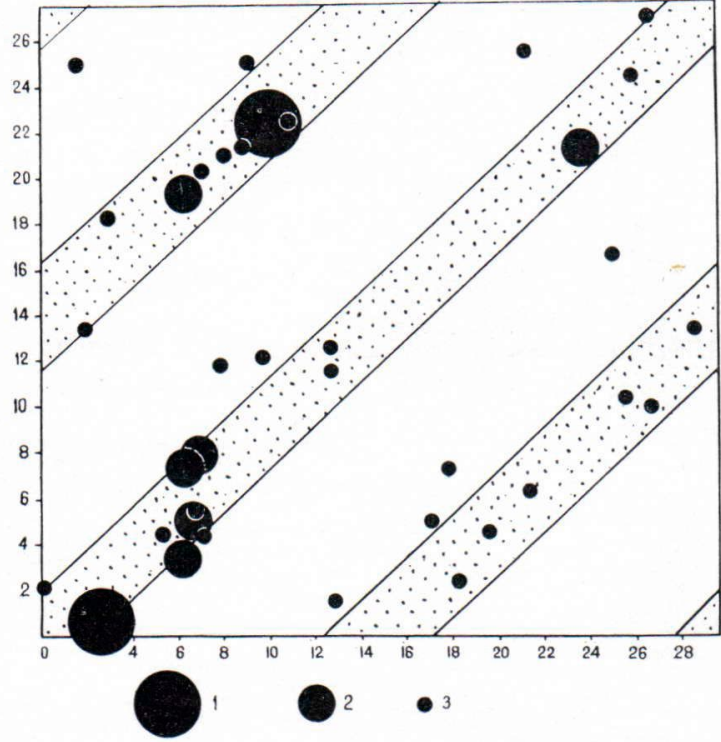

Fig. 4. Distribution of earthquakes of $M \geq 3$ from 1900 - 1950 depending on the a average synodic and reduced anomalistic age. Days of the average synodic month are on the horizontal line. Days of the reduced anomalistic month are on the vertical line. The dots show the time during which the lunar orbit perigee is close to the syzygy zones. Circles desingnate earthquakes. The magnitude (M) of the earthquakes are: 1) $5.1-5.2$; 2) $4.1-$ 5.0 ; 3) $3.0-4.0$.

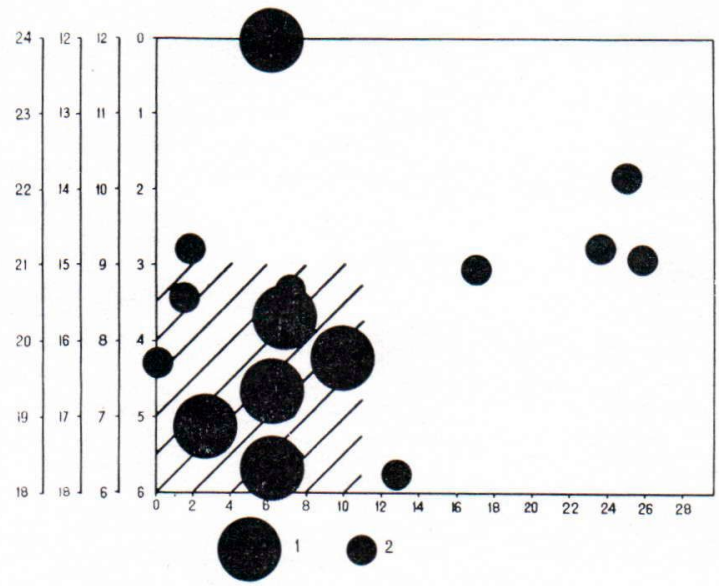

Fig. 5. Distribution of earthquakes in Finland according to the hours of the reduced lunar days (calculated from the moment of the upper culmination of the moon) and simultaneously according to their synodic age (1900-1950).

The magnitude (M) of the earthquakes are: 1) 4.3-5.2; 2) $3.5-4.2$. 
This largely concerns the distribution of earthquakes during the first half of the 20th century.

An earthquake is the result of continuous development within the planet's interior. At the same time specific correlations are presented testifying to the effect of cosmic conditions triggering an energy release in foci. This is of considerable interest.

\section{REFERENCE}

BÅTh, M. (1956). An earthquake Catalogue for Fennoscandia for the years 1891-1950. Stockholm.

Tamrazyan, G. P. (1967). Tide-forming forces and earthquakes. Icarus (International Journal of the Solar System). USA, Vol. 7, No. 1, 59-65.
Tamrazyan, G. P. (1966). Time of origination of earthquakes in the Kuril-Adjoint-to-Kamchatka Region and the local Time (Lunar, Solar). Journal of Physics of the Earth. Japan. Vol. 14, No. 2, 41-48.

Manuscript received, March 15, 1968. 